\title{
Evaluation of demographic, clinical and paraclinical features of children with Guillain-Barré syndrome
}

\author{
Ahad Ghazavi $^{\circledR}$, Ezatolah Abbasi ${ }^{2^{*}}$, Sahar Mohammadi ${ }^{3}$ \\ ${ }^{1}$ Neurophysiology Research Center, Urmia University of Medical Sciences, Urmia, Iran \\ ${ }^{2}$ Department of Pediatric Neurology, Urmia University of Medical Sciences, Urmia, Iran \\ ${ }^{3}$ Urmia University of Medical Sciences, Urmia, Iran
}

Correspondence to:

Ezatolah Abbasi, Email:

Ezatolahabasi1353@gmail.com,

abbasi.e@umsu.ac.ir

Received: 2 August 2020

Accepted: 11 November 2020

ePublished: 23 December 2020

Keywords: Guillain-Barré

syndrome, Polyneuropathy, Acute flaccid paralysis

\begin{abstract}
Introduction: Guillain-Barré syndrome (GBS) is an acute polyneuropathy accompanied by muscle weakness, myalgia, and bulbar involvement.

Objectives: We evaluated demographic, clinical and paraclinical features of children with this disease. Patients and Methods: In this cross-sectional study, the data of 83 patients with GBS were studied. All data about age, gender, place of residence (urban, rural), season of infection, history of infection or vaccination in the last month, clinical demonstrations and spinal magnetic resonance imaging findings with contrast, electromyography (EMG), nerve conduction velocity (NCV), cerebrospinal fluid (CSF), C-reactive protein (CRP) analysis was analyzed.

Results: In this study, the highest incidence of GBS was in the summer $(30.12 \%)$. Regarding clinical symptoms, $54.21 \%$ of patients were unable to walk, and around $14.45 \%$ of cases had positive Gowers' sign. Additionally, $27.71 \%$ of patients reported pain, since $7.22 \%$ of them had severe respiratory distress with the need for mechanical ventilation. In patients who underwent EMG-NCV, findings were in favor of GBS in most cases $(89.83 \%)$. In lumbar puncture, $53 \%$ of patients had normal CSF. Most patients $(75.9 \%)$ had an erythrocyte sedimentation rate (ESR) below $30 \mathrm{~mm} / \mathrm{h}$. CRP of most patients $(83.33 \%$ ) was less than $10 \mathrm{mg} / \mathrm{L}$. Conclusion: The predominant clinical symptoms of patients with GBS are the inability to walk, imbalance, positive Gowers' sign, pain and respiratory distress, since ESR and CRP less than 30 and $10 \mathrm{mg} / \mathrm{L}$ are helpful, respectively.
\end{abstract}

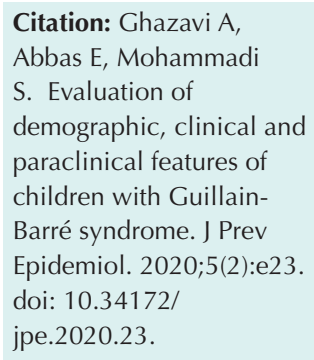

jpe.2020.23.

\section{Introduction}

Guillain-Barré syndrome (GBS) is a postinfection polyneuropathy that mainly affects the motor nerves but sometimes the sensory and autonomic nerves as well (1). GBS is the most common cause of acute flaccid paralysis (2). The overall incidence of GBS in the United States is estimated to be 0.42.4 per 100000 people annually while the incidence of GBS in children is estimated to be $0.1-3.4$ per 100000 children annually (3). Annual incidence in the European population is 1.1 per 100000 annually (4). For every 10 years of age, the incidence of GBS increases by 20\%. The risk of GBS is higher for males than females (5). The overall mortality rate for GBS in the hospitals of the United States is estimated to be $2.58 \%$ (6). However, the overall mortality rate in the European population is reported to be $2.8 \%$ (4). Paralysis usually follows a non-specific viral infection after about 10 days. Muscle weakness usually begins in the lower extremities and progresses to the trunk, upper limbs, and eventually the

\author{
Key point \\ Inability to walk, imbalance, positive Gower's \\ sign, pain and respiratory distress are the dominant \\ symptoms in Guillain-Barré syndrome.
}

bulbar muscles. Sensitivity to touch and muscle pain is common in the early stages of the disease, especially in patients whose disease has started rapidly (1). Examination of cerebrospinal fluid (CSF) is essential for diagnosis. CSF protein is more than twice the normal level in GBS. Sugar is normal and no pleocytosis is seen. The clinical course usually begins within 2-3 weeks. However, involvement of the bulbar and respiratory muscles can lead to death (1). In a study conducted in China, the incidence of GBS in males was higher than in females and in the age group of 1-4 years was higher than in other age groups (7). In another study conducted in Pakistan, the incidence of GBS in males, age group of 11-18 years old and summer was higher (8). 


\section{Objectives}

Given that the incidence of GBS and other epidemiological information related to its differences among races (2-4,611 ), we decided to perform a descriptive study of the condition of children with this disease.

\section{Patients and Methods}

\section{Study design}

This study was a descriptive study performed on 83 patients with GBS. In this study, all cases of patients with this disease who were hospitalized in Shahid Motahari hospital in Urmia from 2010 to 2017 were included. A checklist was prepared based on the goals and guidance of the professors and included age, gender, place of residence (urban, rural), season, and identifiable potential predisposing factors (history of infection or vaccination in the past month and clinical features (pain, ability or inability to move, upper limb involvement, respiratory muscle involvement and need for mechanical ventilation) and paraclinical features consisting of spinal magnetic resonance imaging (spinalMRI) findings with contrast, electromyography (EMG), nerve conduction velocity (NCV), CSF, C-reactive protein (CRP) was analyzed. Patients over the age of 15 years and files without complete information about patients were excluded from the study.

\section{Ethical issues}

Human rights were respected in accordance with the Helsinki Declaration 1975, as revised in 1983. The informed consent was taken from the patients. The study was approved by ethics committee of Urmia University of Medical Science (Ethical cod\# IR.UMSU.REC.1396.175). This study was extracted from medical thesis of Sahar Mohammadi at this university (Thesis \#3262).

\section{Statistical analysis}

All data were collected and entered into SPSS version 21. Descriptive data were presented in frequency (percentage), mean $\pm \mathrm{SD}$, table, and bar chart. $P$ value less than 0.05 was considered significant.

\section{Results}

There were 143 cases of acute flaccid paralysis, of which 83 (58.4\%) were GBS. In this study, 42 (50.6\%) patients were in the age group of 5-10 years. Additionally, 45 patients $(54.21 \%)$ of the patients lived in the urban area and 38 patients $(45.87 \%)$ lived in the rural area. Most patients $(30.12 \%)$ were affected in the summer. There was a history of respiratory or gastrointestinal infection in the past month among 54 patients $(65.6 \%)$, while 29 patients (34.93\%) had no history of previous infection. During the last month, one patient $(1.2 \%)$ was vaccinated and 82 patients (98.79\%) had no history of vaccination (Table 1).

Regarding clinical symptoms, the results showed that 23 patients $(27.71 \%)$ reported myalgia and $60(72.78 \%)$ patients did not have pain. In terms of respiratory involvement and the need for mechanical ventilation, six patients $(7.22 \%)$ found respiratory involvement and the need for mechanical ventilation, and 77 patients $(92.77 \%)$ did not have respiratory involvement. Thirty patients (36.14\%) had upper limb involvement. However, 13 patients $(15.66 \%)$ had a reduction in distal muscle strength and walked with help, 12 patients (14.45\%) had a positive Gower's sign, 13 patients (15.66 \%) had an imbalance and 45 patients (54.21\%) were unable to walk at all (Table 2). In paraclinical assessment, the results showed that out of 83 patients with GBS, spinal-MRI with contrast was performed for 14 spinal-MRI (16.86\%), of which seven patients (50\%) were in favor of GBS. Additionally, EMGNCV was conducted for 59 patients (71.8\%), of whom 53 patients $(89.83 \%)$ reported GBS and six patients $(10.16 \%)$ were normal. Totally, 70 patients $(84.33 \%)$ did not receive lumbar puncture (LP) or did not consent to LP. Thirteen patients (15.66\%) had LP, in which seven patients (53.84\%) had normal CSF and six patients (46.15\%) had GBS (albuminocytologic dissociation), i.e. increased protein and low cell count. Erythrocyte sedimentation rate (ESR) level in 63 patients $(75.9 \%)$ was below 30 and 20 patients (24.9\%) had ESR above $30 \mathrm{~mm} / \mathrm{h}$. CRP patients were checked for 41 patients $(49.39 \%)$, in which 36 patients

Table 1. Frequency distribution of demographic characteristics of the studied patients

\begin{tabular}{llll}
\hline Variable & & No. & $\%$ \\
\hline \multirow{3}{*}{ Age group (y) } & $\geq 5$ & 42 & 50.6 \\
& $5-10$ & 29 & 34.93 \\
Gender & $10-15$ & 12 & 14.45 \\
& Female & 47 & 56.63 \\
History of vaccination & Male & 36 & 43.37 \\
\hline \multirow{3}{*}{ History of infection } & Yes & 1 & 1.2 \\
& No & 82 & 98.79 \\
& Nos & 1 & 1.2 \\
\multirow{2}{*}{ Season } & Spring & 17 & 98.79 \\
& Summer & 25 & 20.48 \\
& Fall & 21 & 30.12 \\
Place of residence & Winter & 20 & 25.30 \\
& Urban & 45 & 24.09 \\
\hline
\end{tabular}

Table 2. Frequency distribution of clinical symptoms of the studied patients

\begin{tabular}{llcc}
\hline Variable & & No. & $\%$ \\
\hline \multirow{2}{*}{ Gender } & Yes & 23 & 27.71 \\
& No & 60 & 72.28 \\
\multirow{2}{*}{ Limb involvement } & Yes & 30 & 36.14 \\
\cline { 2 - 3 } Need to mechanical & No & 53 & 63.85 \\
ventilation & No & 6 & 7.22 \\
\multirow{2}{*}{ Season } & Reduction in the force of distal limbs & 77 & 92.77 \\
& Positive Gower sign & 13 & 15.66 \\
& Imbalance & 13 & 14.45 \\
& Inability to walk & 45 & 54.21 \\
\hline
\end{tabular}


(87.8\%) were negative. CRP was checked for 42 patients (50.6\%), of which 35 patients $(83.33 \%)$ were below 10 and $7 \mathrm{mg} / \mathrm{L}$ patients (16.66\%) were above $10 \mathrm{mg} / \mathrm{L}$ (Table 3). Regarding pain, 23 patients $(27.71 \%)$ reported limb pain and 60 patients $(72.28 \%$ ) had no pain. Regarding respiratory involvement and need for mechanical ventilation, six patients (7.22\%) had respiratory involvement and need for mechanical ventilation and 77 patients $(92.77 \%)$ did not have respiratory involvement. Additionally, 30 patients (36.14\%) had upper limb involvement and 53 cases $(63.85 \%)$ did not have upper limb involvement. Muscle weakness was assessed and 13 patients (15.66\%) had force reduction in distal muscle and walked with assistance, 12 patients $(14.45 \%)$ had positive Gower's sign, 13 patients (15.66\%) had balance disorders and 45 patients (54.21\%) were unable to walk at all (Table 4, Figure 1).

\section{Discussion}

In this study, we examined the demographic characteristics, clinical and paraclinical symptoms of children with GBS. The results of this study are somewhat similar to the results of research conducted in other parts of the world, since there are only a few differences. In this study, the ratio of males to females is 1.3. In the study by Barzegar et al, the ratio was the same (9).

Table 3. Frequency distribution of paraclinical finding of the studied patients

\begin{tabular}{llcc}
\hline Variable & & No. & $\%$ \\
\hline \multirow{2}{*}{ Spinal-MRI } & Normal & 7 & 50 \\
& Indicating GBS & 7 & 50 \\
EMG-NCV & Normal & 53 & 71.08 \\
& Indicating GBS & 6 & 10.16 \\
CSF & Normal & 7 & 53.84 \\
& Indicating GBS & 6 & 46.15 \\
ESR $(\mathrm{mm} / \mathrm{h})$ & $>30$ & 20 & 24.09 \\
& $<30$ & 63 & 75.9 \\
CRP $(\mathrm{mg} / \mathrm{L})$ & $<10$ & 7 & 16.66 \\
& $>10$ & 35 & 83.32 \\
& Negative & 36 & 87.8 \\
CRP results & $1^{+}$ & 3 & 7.31 \\
& $2^{+}$ & 1 & 2.43 \\
& $3^{+}$ & 1 & 2.43 \\
\hline
\end{tabular}

Table 4. Frequency distribution of clinical symptoms of the studied patients

\begin{tabular}{llll}
\hline Clinical symptoms & & No. & \% \\
\hline \multirow{2}{*}{ Pain } & Yes & 27.71 & 23 \\
& No & 72.28 & 60 \\
Respiratory involvement & Yes & 7.22 & 6 \\
and need for & No & 92.77 & 77 \\
mechanical ventilation & Yes & 36.14 & 30 \\
Upper limbs & No & 63.85 & 53 \\
involvement & Force reduction in distal muscles & 15.66 & 13 \\
& Positive Gower sign & 14.45 & 12 \\
Weakness of muscles & Balance disorder & 15.66 & $` 13$ \\
& Inability to move & 54.21 & 45 \\
\hline
\end{tabular}

In the study by Tung et al, the male to female ratio was 2.02 (7). In a study by Jasem et al in Iraq (11), Sladky et al in Pakistan (8) and Molinero et al in Honduras (13), the incidence of GBS in males was higher. According to a research conducted by Sejvar et al, the risk of GBS is higher for males than females (5). According to the results obtained in terms of age distribution, GBS has the highest percentage in the age group under five years (50.6\%) which is consistent with the study by Tung Jun et al (7) as well as the study by Jasem et al (11), while in the study by Sladky et al (8), the disease was more in the age group of 11-18 years, which is inconsistent with the results of our study. According to previous studies, for every 10 years of aging, the incidence of GBS increases by $20 \%$ (5).

In the present study, the rate of infection in urban dwellers was higher than (54.21\%) in rural dwellers (45.87\%). In the study by Molinero et al, the incidence of GBS in rural areas was slightly higher (13). Additionally, in the study of Jasem et al, the incidence of GBS was higher in rural areas (11), which is contradicting the results of our study. In the present study, the highest incidence of GBS occurred in the summer (30.12\%), while in the study by Sladky et al, the prevalence of GBS in the summer was higher than in other seasons (8). In the study by Momen et al, most of the patients detected in the fall (2) while in the study by Barzegar et al, patients were more likely to have GBS in the winter (9), which is contrary to the results of our study too. The higher incidence of GBS in the summer in our region can be justified by the high prevalence of infectious (possibly gastrointestinal) diseases.

In our study, most patients reported a history of respiratory or gastrointestinal infection in the past month. In a study by Tang et al, $46.1 \%$ of patients reported a history of infection in the last 1 to 4 weeks of admission (7). In the present study, the most common clinical symptom was muscle weakness in all patients. Most of the patients were unable to walk at all, fewer walked with help; a group had a positive Gower's sign, and imbalance. Most patients did not report pain $(72.88 \%)$ and did not experience respiratory involvement and the need for mechanical ventilation. In the study by Devos et al, walking and pain disorders were the most common symptoms (14). In the study by Tang et al, $9.5 \%$ of patients needed mechanical ventilation (7). In MRI, half of the cases reported GBS and the other half were normal. In a study by Yikilmaz et al, all patients except two patients had thickening and contrast inhalation in nerve roots and cauda equina in spinal-MRI (15). Regarding EMG-NCV, in most cases, EMG-NCV findings were in favor of GBS (89.83\%), which was reported to be abnormal in the study by Devos et al, even during the first week of electrophysiological studies (14). In a study by Yikilmaz et al, of 28 patients tested with the EMG-NCV, 25 patients were in favor of GBS (15). Their normalcy was probably due to the fact that all of them were performed during the first week of the illness.

In our study, $53 \%$ of patients with LP had normal CSF 


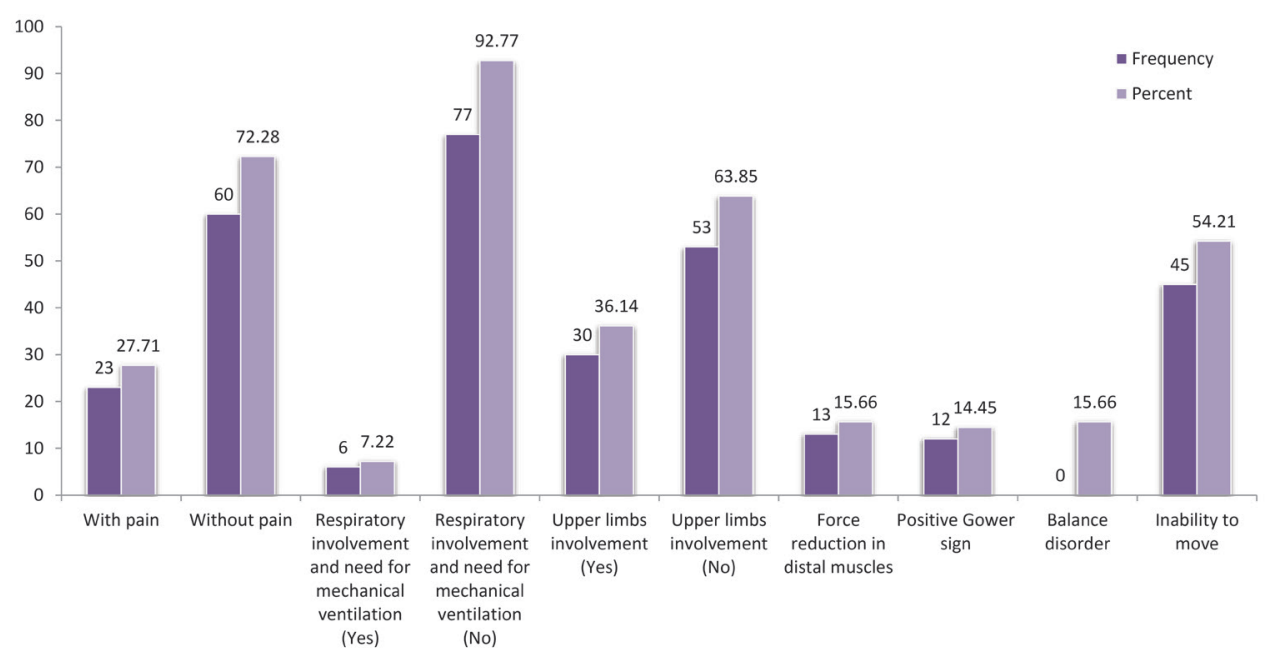

Figure 1. Frequency distribution of clinical symptoms of the studied patients.

and $46 \%$ had CSF in favor of GBS. In the study by Tang et al, $88 \%$ of patients had cytoalbuminologic dissociation in patients undergoing lumbar puncture. In the study by Yikilmaz et al, cytoalbuminologic dissociation was reported in 37 patients, most patients (75.9\%) had an ESR below $30(\mathrm{~mm} / \mathrm{h})$. CRP was also negative in most patients $(83.33 \%)$ below $10(\mathrm{mg} / \mathrm{L})$. Given that GBS is a non-inflammatory disease, inflammatory indicators such as ESR and CRP are not expected to increase.

\section{Conclusion}

The most common age, gender and season for GuillainBarré disease is 1 to 5 years, males, and summer, respectively. The predominant clinical symptoms of patients with GBS are the inability to walk, imbalance, positive Gower's sign, pain and respiratory distress. CSF and spinal-MRI detection cannot help much. ESR and CRP less than $30 \mathrm{~mm} / \mathrm{hr}$ and 10 are helpful, respectively.

\section{Limitations of the study}

In this study, incomplete and illegible information of hospitalized patient files was one of the limitations in achieving the desired results. Some information about the history and course of the disease was inconsistent, and some of the information in the files was not verified. Besides, some patients were discharged with personal consent before completing the diagnostic and treatment procedures, which were removed from the study and the number of samples was reduced.

\section{Authors' contribution}

AG, EA and SM were the principal investigators of the study. AG and EA were included in preparing the concept and design. SM and EA revisited the manuscript and critically evaluated the intellectual contents. All authors participated in preparing the final draft of the manuscript, revised the manuscript and critically evaluated the intellectual contents. All authors have read, approved the content of the manuscript and confirmed the accuracy or integrity of any part of the work.
Ethical considerations

Ethical issues (including plagiarism, data fabrication, double publication) have been completely observed by the authors.

\section{Competing interests}

The authors declare that they have no competing interests.

\section{Funding/Support}

This article was taken from medical thesis of Sahar Mohammadi, at Urmia University of Medical Sciences (Grant \#3262). The authors would like to thank the vice chancellor of research deputy of Urmia University of Medical Sciences for financial support.

\section{References}

1. Ryan MM. Pediatric Guillain-Barrésyndrome. Curr Opin Pediatr. 2013;25(6):689-93. doi: 10.1097/MOP.0b013e328365ad3f.

2. Momen AA, Shakurnia A. The Epidemiology of Guillain-Barré Syndrome in Children under 15 Years Old in Southwest Iran. Biomed Hub. 2017;2:1-8. doi: 10.1159/000480693.

3. Rosen BA. Guillain-Barré syndrome. Pediatr Rev. 2012;33:16470; quiz 170-1. doi: 10.1542/pir.33-4-164.

4. Chroni E, Papapetropoulos S, Gioldasis G, Ellul J, Diamadopoulos N, Papapetropoulos T. Guillain-Barré syndrome in Greece: seasonality and other clinicoepidemiological features. Eur J Neurol. 2004;11:383-8. doi: 10.1111/j.1468-1331.2004.00799.x.

5. Sejvar JJ, Baughman AL, Wise M, Morgan OW. Population incidence of Guillain-Barré syndrome: a systematic review and meta-analysis. Neuroepidemiology. 2011;36:123-33. doi: 10.1159/000324710.

6. Alshekhlee A, Hussain Z, Sultan B, Katirji B. GuillainBarré syndrome: incidence and mortality rates in US hospitals. Neurology. 2008;70:1608-13. doi: 10.1212/01. wnl.0000310983.38724.d4.

7. Tang J, Dai Y, Li M, Cheng M, Hong S, Jiang L, Cai F, Zhong M. Guillain-Barré syndrome in Chinese children: a retrospective analysis. Pediatr Neurol. 2011;45:233-7. doi: 10.1016/j. pediatrneurol.2011.06.007.

8. Sladky JT. Guillain-Barre syndrome in children. J child Neurol. 2004 Mar;19(3):191-200.

9. Barzegar M, Dastgiri S, Karegarmaher MH, Varshochiani A. Epidemiology of childhood Guillan-Barre syndrome 
in the north west of Iran. BMC Neurol. 2007;7:22. doi: 10.1186/1471-2377-7-22.

10. Barzegar M, Jalali Z, TopchizadehV. Epidemiological, clinical andelectrodiagnostic findings in childhood Guillain-Barré syndrome. Med J Islam Repub Iran. 2003;17:123-127.

11. Jasem J, Marof K, Nawar A, Khalaf Y, Aswad S, Hamdani F, Islam M, Kalil A. Guillain-Barré syndrome as a cause of acute flaccid paralysis in Iraqi children: a result of 15 years of nationwide study. BMC Neurol. 2013;13:195. doi: 10.1186/14712377-13-195.

12. Vandoorn PA, Ruts L, Jacobs BC. Clinical features, pathogenesis, and treatment of Guillain-Barré syndrome. Lancet Neurol. 2008;7:939-50. doi: 10.1016/S1474-4422(08)70215-1.

13. Molinero MR, Varon D, Holden KR, Sladky JT, Molina
IB, Cleaves F. Epidemiology of childhood GuillainBarré syndrome as a cause of acute flaccid paralysis in Honduras: 1989-1999. J Child Neurol. 2003;18:741-7. doi: 10.1177/08830738030180110801.

14. Devos D, Magot A, Perrier-Boeswillwald J, Fayet G, LeclairVisonneau L, Ollivier Y, et al. Guillain-Barré syndrome during childhood: particular clinical and electrophysiological features. Muscle Nerve. 2013;48:247-51. doi: 10.1002/ mus.23749.

15. Yikilmaz A, Doganay S, Gumus H, Per H, Kumandas S, Coskun A. Magnetic resonance imaging of childhood Guillain-Barre syndrome. Childs Nerv Syst. 2010;26(8):1103-8. doi: 10.1007/ s00381-010-1197-8. 